\title{
Simulations of electrostatic modes of non-neutral plasmas with small aspect ratio in a Penning trap
}

Grant W. Mason

masong@utah-inter.net

Ross L. Spencer

ross_spencer@byu.edu

Jonathan A. Bennett

Follow this and additional works at: https://scholarsarchive.byu.edu/facpub

Part of the Astrophysics and Astronomy Commons, and the Physics Commons

\section{Original Publication Citation}

Mason, Grant W., Ross L. Spencer, and Jonathan A. Bennett."Simulations of electrostatic modes of non-neutral plasmas with small aspect ratio in a Penning trap." Physics of Plasmas 3 (1996): 152-1511.

\section{BYU ScholarsArchive Citation}

Mason, Grant W.; Spencer, Ross L.; and Bennett, Jonathan A., "Simulations of electrostatic modes of nonneutral plasmas with small aspect ratio in a Penning trap" (1996). Faculty Publications. 677.

https://scholarsarchive.byu.edu/facpub/677

This Peer-Reviewed Article is brought to you for free and open access by BYU ScholarsArchive. It has been accepted for inclusion in Faculty Publications by an authorized administrator of BYU ScholarsArchive. For more information, please contact ellen_amatangelo@byu.edu. 


\title{
Simulations of electrostatic modes of non-neutral plasmas with small aspect ratio in a Penning trap
}

\author{
Grant W. Mason, Ross L. Spencer, and Jonathan A. Bennett \\ Department of Physics and Astronomy, Brigham Young University, Provo, Utah 84602
}

(Received 3 November 1995; accepted 1 February 1996)

\begin{abstract}
The dependence on induced charge, experimental geometry, and temperature of electrostatic modes in very low aspect ratio non-neutral plasmas in a Penning trap is considered. The modes are of interest as non-destructive diagnostics of the shape of the plasmas. These investigations include equilibrium calculations of plasma shapes and profiles at finite temperature and particle-in-cell simulations of axisymmetric modes. The results of the simulations are compared to the zero-temperature theory by Dubin [Phys. Rev. Lett. 66, 2076 (1991)] taken to first-order in the aspect ratio and to experimental measurements by Weimer et al. [Phys. Rev. A 49, 3842 (1994)]. In general, it is concluded that the Dubin theory provides a means to obtain reasonable estimates of plasma parameters, including density, radius, and axial length, for plasmas in the very important regime for which the axial length is comparable to the Debye length. In addition, dependence on induced charge, equilibrium shape, and plasma temperature are identified which can likely be used to improve agreement between theory and experiment. (C) 1996 American Institute of Physics. [S1070-664X(96)02105-6]
\end{abstract}

\section{INTRODUCTION}

Penning traps such as the one shown in Fig. 1 use a combination of electrostatic and magnetic fields in cylindrical geometry to confine one or more charged particles for extended periods of time. Recently Gabrielse et al. ${ }^{1}$ have used such a trap to confine an individual antiproton and measure its cyclotron frequency in a known magnetic field as a sensitive test of the charge-parity-time reversal (CPT) theorem for baryonic matter. In some uses of Penning traps, large enough numbers of particles are confined so that one has the coherent behavior that characterizes a non-neutral plasma. For example, Greaves and Surko ${ }^{2}$ have begun experiments in which they bombard a non-neutral positron plasma confined in a Penning trap with an electron beam to do studies that have potential relevance to positron-electron plasmas in astrophysics. Others confine ions for experiments to study ionion collisions, recombination rates and collisions between ions and neutral atoms, etc. ${ }^{3}$

In some experiments it is desirable to have a nondestructive method of measuring the density, rotation frequency, and shape of the plasma. For example, such information would be particularly important if the plasma were being used as a target in collision experiments. In principle this might be done by measuring the electrostatic mode frequencies of the plasma as the motion induces charge oscillations in the walls of the trap and then comparing the frequencies to theoretical relationships which depend on the desired, but unknown, characteristics of the plasma. This program has recently been given considerable impetus by the work of Dubin ${ }^{4}$ who has calculated the global modes for a spheroidal, zerotemperature non-neutral plasma for which the confining walls of the trap are remote.

Tinkle et $a l .{ }^{5}$ have demonstrated that the frequency of the quadrupole mode of a spheroidal single-component electron plasma in a Penning trap has well-characterized dependences on the plasma aspect ratio, length, and temperature. Excellent agreement between numerical simulations of the plasma and the experiment demonstrates that the simulations are a valuable tool for the detailed study of the modes. Weimer et al. ${ }^{6}$ Bollinger et al., ${ }^{7}$ and Heinzen et al. ${ }^{8}$ have also used measured frequencies and the model of Dubin to calculate density and aspect ratio of electron and ion plasmas at low temperature and to study the evolution of shape and size of the plasma with time.

This paper is primarily an attempt through numerical modeling to better understand certain discrepancies between experiment and the Dubin theory that arose in the work by Weimer et al. $^{6}$ In the Weimer experiments the plasma takes the shape of a thin axisymmetric pancake. In these and other Penning trap experiments in which particles are stored for long periods of time, torques on the plasma system (probably from field asymmetries) will cause the plasma to expand radially into a pancake shape. The only atomic physics experiments where long-lived plasmas are known not to evolve to this shape are laser cooling experiments where laser torques are used to spin up the plasma. Thus the pancake regime explored by Weimer is particularly important to diagnose and understand.

In Sec. II we review the elements of the theory of Dubin which makes predictions relevant to the experiments of Weimer et al. and which were used in interpreting their experimental measurements. In Sec. III we will describe the experiment and the discrepancy with theory and identify factors on which it might depend. In Sec. IV we will describe the simulation methods used to do numerical experiments that might indicate the source of the discrepancy. In Sec. V we will describe the results of numerical experiments that we performed and, finally, in Sec. VI we will draw conclusions from our work.

\section{THEORY}

In the theory of Dubin, a zero-temperature non-neutral plasma is confined axially in cylindrical geometry by an electrostatic potential of the form 


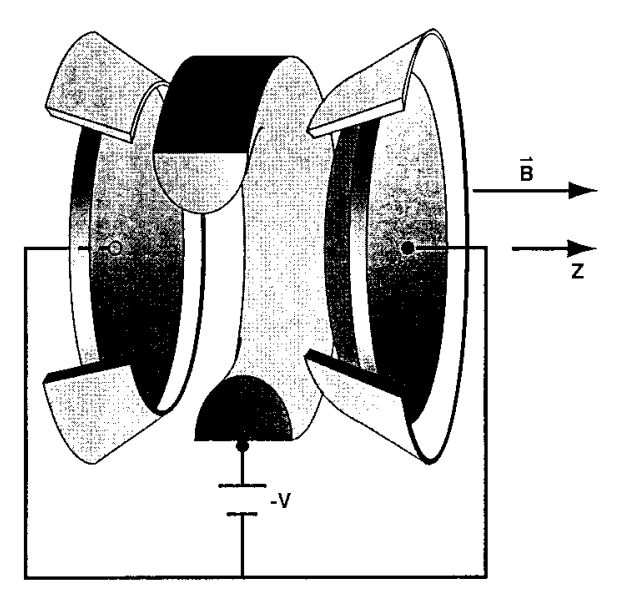

FIG. 1. Penning Trap. The trap produces an approximation to an electrostatic confining potential well of the form $\phi=C\left(z^{2}-r^{2} / 2\right)$ by applying a voltage difference between the endcaps (right and left) and the ring (middle). A potential is applied to the guard ring to tune the confining properties of the trap. Charged particles are confined in the radial direction (vertical) by a uniform horizontal magnetic field.

$$
\phi(r, z)=C\left(z^{2}-r^{2} / 2\right) \text {. }
$$

The plasma in such an ideal well assumes a spheroidal shape. The continuity, momentum, and Poisson's equations are combined to predict a family of global oscillations which includes axisymmetric $(m=0)$ modes and non-axisymmetric $(m \neq 0)$ modes. The mode potentials are expressed as products of associated Legendre functions $P_{l}^{m}$ and $Q_{l}^{m}$ and the factor $e^{i(m \phi-\omega t)}$, where $\phi$ is the azimuthal angle about the trap axis and $\omega$ is the mode frequency. The integer $l$ is related to $n_{z}$, the number of axial nodes at $r=0$, and $n_{r}$, the number of radial nodes in the midplane, by the relationship $l=n_{z}+2 n_{r}$. The mode $(l, m)=(1,0)$ is the axial center-ofmass oscillation of the plasma with frequency $\omega_{z}$.

The expressions that follow from the Dubin theory depend on the aspect ratio $\alpha$ of the plasma which is defined to be the ratio of the half-length of the plasma to its radius. In the zero-temperature theory, the plasma is sharp-edged and the aspect ratio is well-defined. For thin plasmas in which $\alpha$ is small, the frequency expressions may be reduced to a first-order approximation in the aspect ratio resulting in a series of relationships for each of several modes. Experimentally, the plasmas of interest here have the aspect ratio of a thin pancake $(\alpha \approx 0.01)$ resembling a drumhead. This "drumhead" may vibrate with both axisymmetric and nonaxisymmetric modes and both types are measured in the Weimer et al. experiment, although the work described here treats only the axisymmetric case $m=0$. For the modes of interest here, the approximate relationships are

$$
\begin{aligned}
& \left|\omega_{1,0}\right|=\omega_{z}, \\
& \left|\omega_{3,0}\right|=\omega_{z}\left[1-\frac{5}{16} \pi \alpha\right], \\
& \left|\omega_{5,0}\right|=\omega_{z}\left[1-\frac{161}{256} \pi \alpha\right],
\end{aligned}
$$

$$
\left|\omega_{7,0}\right|=\omega_{z}\left[1-\frac{969}{1024} \pi \alpha\right] .
$$

For small aspect ratios $(0.01$ or less), these approximations are precise to about $0.01 \%$ [having dropped terms of order $\alpha^{2} \approx(0.01)^{2}$ ] when used to determine frequencies but are less precise (about $2 \%$ ) when used to solve for $\alpha$, since it depends on a difference between frequencies which is small compared to the magnitude of the frequencies themselves. The frequency $\omega_{z}$ in these equations is the frequency of the axial center-of-mass motion of the plasma and is determined by the charge and mass of the confined particles, the geometry, and the external electrical potentials of the trap. For an ideal trap as assumed by Dubin, this frequency is the same for particles at any radius within the trap and is independent of the amplitude of the oscillation of the particles.

The Dubin theory also predicts radial profiles of the axial displacement for the modes of the spheroidal plasmas. Defining $x$ to be the ratio of radius within the plasma, $r$, to the plasma radius, $r_{p}$, the modes have shapes (in the limit $z \rightarrow 0$ ) given by ${ }^{4}$

$$
\delta z(x)_{(l, 0)} \propto \frac{P_{l}\left(\sqrt{1-x^{2}}\right)}{\sqrt{1-x^{2}}},
$$

where $P_{l}$ are the set of Legendre polynomials. Specifically,

$$
\begin{aligned}
& \delta z(x)_{(3,0)} \propto\left(1-2.5 x^{2}\right), \\
& \delta z(x)_{(5,0)} \propto\left(1-7 x^{2}+7.875 x^{4}\right), \\
& \delta z(x)_{(7,0)} \propto\left(1-13.5 x^{2}+37.12 x^{4}-26.81 x^{6}\right),
\end{aligned}
$$

where $\delta z(x)$ is the axial displacement of the pancake as a function of radius for the indicated mode.

Finally, to first order in $\alpha$, the theory relates the plasma frequency, $\omega_{p}$, to $\omega_{z}$ and $\alpha$,

$$
\omega_{p}=\omega_{z}\left(1+\frac{1}{4} \pi \alpha\right) .
$$

Thus, if the aspect ratio can be obtained, one can obtain the plasma density from the plasma frequency. Knowing the particle number and assuming uniform density and spheroidal shape, one can then obtain the radius, $r_{p}$, and thickness, $z_{p}$, of the plasma.

\section{EXPERIMENT}

The experimental situation to which the theory is to be applied differs from the ideal theoretical situation in several respects. Even though experiments may be performed at low temperatures, the densities are also low and the plasma is thin leading to Debye lengths that are comparable to the thickness of the plasmas. Hence, temperature effects may be present. The walls of the trap are not infinitely far away, so induced charges may be important. The magnetic field is not exactly parallel to the trap axis nor does the trap necessarily have ideal cylindrical symmetry. And finally, the electrostatic field that provides the harmonic well of the trap does not give the ideal form of Eq. (1) so that even the axial particle bounce frequency may have both amplitude and radial dependence. Thus, there are several potential sources of discrepancy between theory and experiment which may limit 
the degree to which useful diagnostic information can be extracted from the measurement of global mode frequencies.

In the experiments of Weimer et al., cryogenic cooling was used to reduce the electron temperature to close to $4 \mathrm{~K}$. Magnetron sideband cooling was then used to stabilize and compress the electron cloud. Over long times (3000 s) the trapped plasmas were then observed to undergo a radial expansion which decreases the aspect ratio of the plasma and eventually leads to loss of confinement. Since the total canonical angular momentum of the system is conserved in the absence of torques, ${ }^{9}$ the radial expansion and consequent loss of plasma can be interpreted as evidence for torques for which the likely cause is asymmetries in the electric or magnetic fields of the trap. The trap itself is only about one centimeter in diameter and consists of machined parts that only approximate the surfaces of a hyperboloid. To compensate, the trap is provided with a guard ring of variable potential which can be used to tune the trap to more nearly ideal harmonic conditions, but it is still impossible to tune the trap everywhere simultaneously.

In practice the trap was tuned by confining a small number of electrons near the origin, exciting their axial centerof-mass motion to increasing amplitudes, and adjusting the guard ring voltage to eliminate amplitude dependence of the frequency of the mode, i.e. to make the well ideal, at least in the region near the origin. This tuning process is a local rather than a global tuning process. In the experiments of Weimer et al., tuning was accomplished with a guard ring voltage of $+6.3 \mathrm{~V}$. However, the detection of the modes was improved (for unknown reasons) when detuned to $+2.9 \mathrm{~V}$ and it was at this voltage that the data shown in Fig. 5 of Weimer et al. were taken. ${ }^{10}$ The mode frequencies leading to the figure were measured with an estimated precision of about $0.01 \% .^{10}$

Figure 5 of Weimer et al. ${ }^{6}$ shows a plot over time of the changing aspect ratio of an electron plasma in the trap as computed from Eqs. (2)-(5). However, at each point in time, different values of the aspect ratio emerge from the Dubin equations for the different modes, with a fairly constant spread over time of $20-25 \%$. The predicted aspect ratios are monotonically decreasing with increasing number of radial nodes. Since the equations themselves are thought to be precise to within at least $2 \%$, the spread seems to be anomalous. In what follows, we investigate by numerical experiments some possible causes of the spread.

\section{SIMULATION}

To investigate the behavior of the plasmas via simulation, we first compute equilibria using a Poisson-Boltzmann code. ${ }^{11}$ The code allows us to approximate the interior surfaces of the trap, including the guard ring. Figure 2 shows a typical configuration of plasma and details of the trap. The endcaps (right and left ends) are held at $-10.45 \mathrm{~V}$, the ring (top) is held at $0.0 \mathrm{~V}$, and the guard ring is set at selected values, $V_{g}$, to compare tuned and detuned traps. The computing region is sufficiently large $(1.37 \mathrm{~cm}$ axially and 0.8 $\mathrm{cm}$ radially) so that details of the endcap, ring, and guard

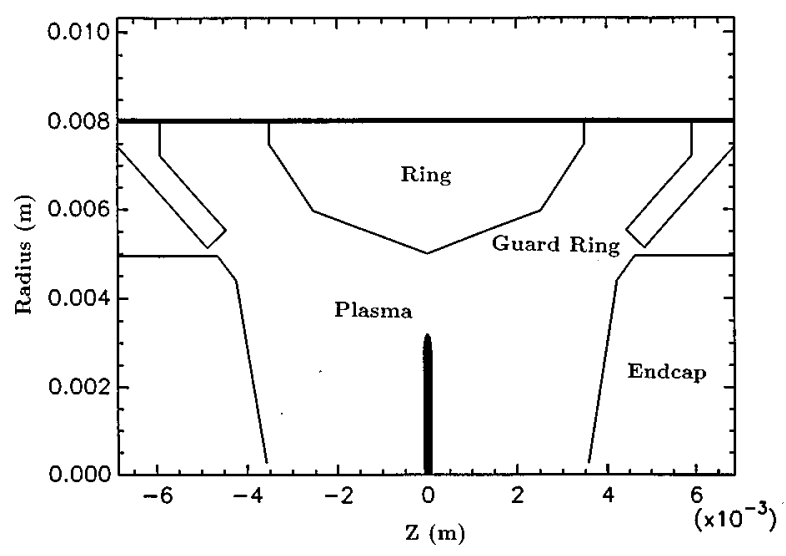

FIG. 2. Simulated Penning Trap. The endcaps (right and left), ring (top) and guard rings are modelled by line segments held at the constant potentials of the Weimer experiment. In both the model and the actual experiment, the surfaces are straight as shown. The lengths of the lines in the model are faithful to the length of the cuts in the experimental trap. The plasma is the vertical structure in the center of the diagram. Observe that for this longer plasma, the width of the plasma increases with radius. The numerical model has azimuthal symmetry about the horizontal axis at the bottom of the figure.

ring are simulated. The ring has an inner radius of $0.5 \mathrm{~cm}$. The point of closest axial proximity of the endcap to the origin is $0.35 \mathrm{~cm}$.

The plasmas considered here are strongly magnetized by a constant magnetic field along the axis of the trap so that the axial and radial motions of the electrons are decoupled. The Poisson-Boltzmann equilibrium code solves nonlinear Poisson equations of the form,

$$
\nabla^{2} \phi=-\frac{q}{\epsilon} n(r, \phi) \text {. }
$$

The code can be used to solve for either equilibria with a specified midplane density profile or for self-consistent thermal equilibria ${ }^{12}$ with specified values of central density and radial extent corresponding to some fixed canonical angular momentum. Because the plasmas in the experiments were so long lived that global thermal equilibrium might have been approached, and because there was no experimental information about radial profiles, we thought it reasonable to assume that the plasma was in global thermal equilibrium. In order to facilitate comparison with Dubin's theory and with the experiments, the equilibrium calculation was run in such a way (unless otherwise indicated) that the central density and the plasma radius were adjusted during the iteration process to give a fixed number of electrons $(43,000$ as reported by Weimer et $\left.a l .{ }^{6}\right)$ and also to give the same canonical angular momentum as a cold spheroid with a chosen plasma radius and aspect ratio. This procedure is equivalent to the physically-realizable process of heating a cold spheroid without applying any torque to it.

Equilibria thus calculated are provided as the starting point for a particle-in-cell (PIC) simulation of the plasma within the trap. The code is faithful to the geometry and plasma characteristics established in the equilibrium code. In this $r-z$ simulation, particles are tracked in a twodimensional phase space $\left(z, v_{z}\right)$. Since the plasma is axisym- 


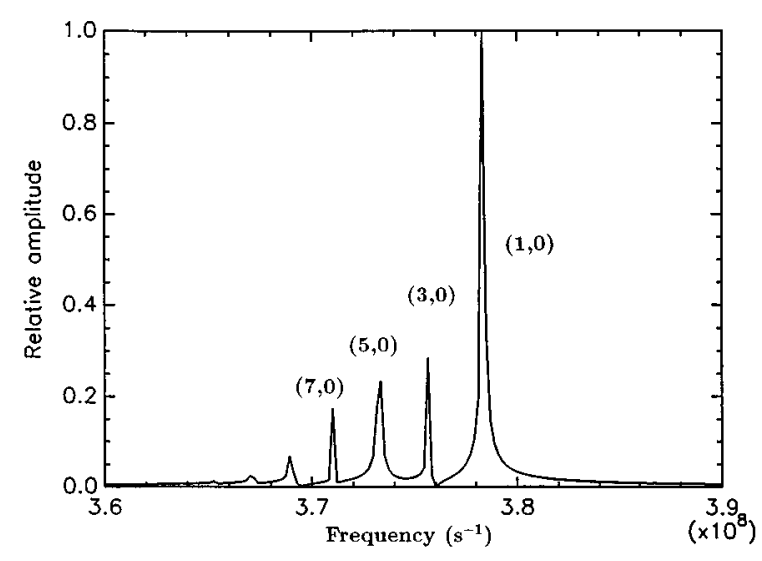

FIG. 3. Mode Spectrum from PIC code. The time sequence analyzed is the motion of the center-of-mass of the plasma. The prominent peak is the frequency of the axial center-of-mass motion of the plasma, $(1,0)$. The less prominent peaks are the $(3,0),(5,0),(7,0)$, and $(9,0)$ modes ordered from right to left. In this case the guard ring voltage of the trap is $+6.3 \mathrm{~V}$ and the radius of the plasma is about $3.2 \mathrm{~mm}$.

metric and drift motion is assumed in the plane perpendicular to the confining magnetic field, the $r$-coordinates of the particles never change once they are loaded. The PIC simulation assumes the same ideal cylindrical symmetry and, hence, computes mode frequencies for a plasma with stationary radial profile.

Although precise radial mode structures are not known for the geometry of the experiment, we use the known mode structures from the ideal Dubin theory [Eqs. (7)-(9)] to seed axisymmetric modes of the simulated plasma. Time sequences that track the center of mass motion as well as the overlap integrals of the density-weighted axial displacement of the plasma with the seeding functions are produced and Fourier analyzed to yield mode frequencies. Lorentzian fits to the peaks of the spectrum are used to refine the precision of the mode frequencies. A typical spectrum of modes is shown in Fig. 3. Aspect ratios are computed from Eqs. (2)(5) for such modes and compared to see if the anomalous spreading observed in the experiments can be observed as conditions in the simulation are varied.

Because we are using a grid ( 80 radial $\times 500$ axial $)$ to simulate the plasma and because we want to represent the effects of the geometry of the experiment surrounding the plasma, we are limited by our computer memory resources to grids that can resolve the plasma thickness when the aspect ratios are on the order of 0.01 or larger. This corresponds to about a half-dozen grid cells across the thin dimension of the plasma. Experimentally, the data begin at aspect ratios near to this value, but the plasmas then evolve to become progressively thinner until the aspect ratio is about 0.002 . Thus, we are not able to simulate the experiment over its full range of aspect ratios. In any case, we simulate the plasma for a single choice of aspect ratio at a time. No attempt is made to follow the evolution of the aspect ratio of the plasma.

In order to obtain sufficient precision in the frequencies of the simulated modes, we take 131072 time steps of onequarter nanosecond each. Based on these parameters, the spectral peaks should be localized to within

$$
\frac{\Delta \omega}{\omega}=\frac{2 \pi}{\omega N \Delta t} .
$$

Allowing for an improvement factor of $1 / 3$ obtained by fitting a Lorentzian to the peaks, we obtain an estimated precision, $\Delta \omega / \omega \approx 0.02 \%$. This agrees with our empirical observation that the numerical simulations reproduce the mode frequencies roughly to about 5 parts in 38000 when started with different initial seeding perturbations. As noted in Sec. II, Eqs. (2)-(5) then yield the aspect ratio with a precision of about $2-3 \%$.

\section{NUMERICAL EXPERIMENTS}

In this section we describe numerical experiments with simulated plasmas designed to measure the extent to which induced charge, realistic geometry, and temperature affect the measurement of aspect ratios via Eqs. (2)-(5). For purposes of defining the aspect ratio, we measure the radial and axial lengths of the plasma to the point at which the density falls to half-maximum.

We first turn our attention to the possible effect on the mode frequencies of induced charge in the conducting surfaces of the trap. In the Appendix we estimate the effects of image charge using electrostatics in the complex plane. The calculation indicates that the major effect on mode frequencies of induced charge in either the ring or the endcaps is to lower the frequency of the center-of-mass mode preferentially, with decreasing relative shifts as the mode number increases. The shifts in frequency from this effect for plasmas with a uniform density profile translate into a narrowing of spread or actual reversal of the ordering of the aspect ratios as they emerge from Eqs. (2)-(5) when compared to the experimental data of Weimer et al.

We then performed numerical experiments to test this conclusion using equilibria which were not in global thermal equilibrium, but rather equilibria which were constrained to have a flat radial density distribution similar to the uniform density distributions treated in the Appendix. We estimated the effect of induced charges on the predicted aspect ratios by creating an equilibrium at $4 \mathrm{~K}$ with a central density of $1.57 \times 10^{13} / \mathrm{m}^{3}$, a radial extent of $3.6 \mathrm{~mm}$ and an aspect ratio of 0.0090 in an ideal hyperbolic well (not the trap of Fig. 2). Its axial and radial extent and confining voltages were comparable to those of the Weimer experimental trap. We then froze the values of the potential at the boundaries of the computing region (at horizontal positions $z= \pm 3.5 \mathrm{~mm}$ ) as obtained in the equilibrium determination to serve as boundary conditions for the PIC simulation and obtained mode frequencies for $(1,0),(3,0)$, and $(7,0)$. [The $(5,0)$ mode was not seeded in these numerical experiments since we were interested in the spread between $(3,0)$ and $(7,0)$.] We then repeated the entire process with the right-left boundaries moved inward to $z= \pm 2.5 \mathrm{~mm}$.

The results of the simulations were consistent with the predictions of the calculation in the Appendix. Moving the walls closer to the plasma to increase the induced charge effect reduced the center-of-mass mode frequency by 10 parts in 35,000 (significant at our level of precision), but shifted the $(3,0)$ and $(7,0)$ modes downward by less than half 


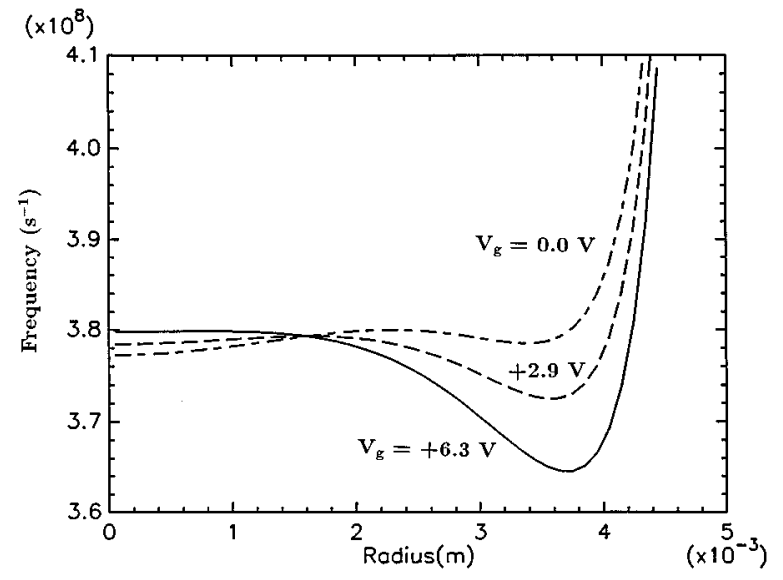

FIG. 4. Vacuum Penning frequency profiles as a function of radius. As the guard ring voltage is increased, the vacuum Penning frequency becomes a function of radius. The solid curve is for a guard ring voltage of $+6.3 \mathrm{~V}$. The curve for $+2.9 \mathrm{~V}$ is bracketed by the curves for $0.0 \mathrm{~V}$ and $+6.3 \mathrm{~V}$. Observe that the vertical scale is truncated so that the differences are exaggerated.

of this amount, an amount which is not significant (borderline) at our level of precision. The shifts produce aspect ratios from Eqs. (2)-(5) which are actually reversed in order from the experimental values, i.e. increasing aspect ratio rather than decreasing with increasing mode number. Although not insignificant, the effect of the induced charge works in the wrong direction to explain the spreading effect observed in the experimental data.

We now turn to a more "realistic" set of simulations in which we replace the ideal hyperbolic confining well with a well created by an endcap, ring, and guard ring such as is shown in Fig. 2. In Fig. 4 we show the radial profile of the axial center-of-mass mode frequency that a small test charge would experience in the trap without any other electrons. We refer to this purely geometric characteristic of the well as the vacuum Penning frequency profile. In the ideal geometry

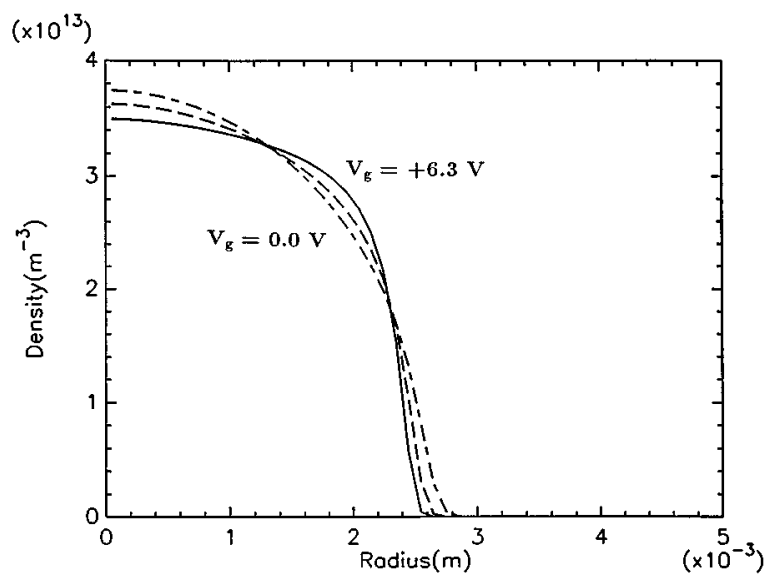

FIG. 5. Midplane density profiles. The profiles shown are for plasmas of shorter radial extent $(\alpha=0.019-0.022)$ where the effect of the nonideal shape of the ring and the effect of the guard ring is less pronounced. The solid curve is for a guard ring voltage of $+6.3 \mathrm{~V}$. The curve for $+2.9 \mathrm{~V}$ is bracketed by the curves for $0.0 \mathrm{~V}$ and $+6.3 \mathrm{~V}$.

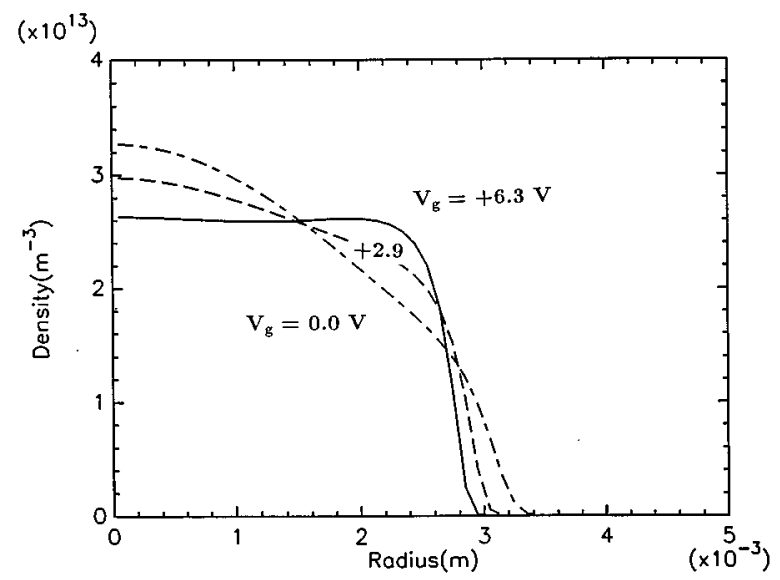

FIG. 6. Midplane density profiles. The profiles shown are for plasmas of medium radial extent ( $\alpha=0.014-0.017)$. The solid curve is for a guard ring voltage of $+6.3 \mathrm{~V}$. The curve for $+2.9 \mathrm{~V}$ is bracketed by the curves for 0.0 $\mathrm{V}$ and $+6.3 \mathrm{~V}$

used in the induced charge simulations just discussed, this profile is constant as a function of radius. In more realistic geometry, however, the profile depends on radius and on the guard voltage. Figure 4 shows profiles for three choices of guard ring voltage: $0.0,+2.9$, and $+6.3 \mathrm{~V}$. Observe that the scale of the vertical axis is truncated so that the differences are exaggerated.

One observes that the profiles are significantly different at the level of precision of both experiment and simulations. Rather than having a single axial center-of-mass mode frequency, for example, plasmas that extend beyond $2.0 \mathrm{~mm}$ must adjust to some new common frequency. From Fig. 4 it appears that plasmas that have radial extent less than $2.0 \mathrm{~mm}$ would find the trap that is tuned with a guard voltage of +6.3 $\mathrm{V}$ to be an ideal trap, since the profile for this voltage is most nearly flat near the origin. Further, if the trap were tuned by observing the behavior of electrons near $r=0$, one would

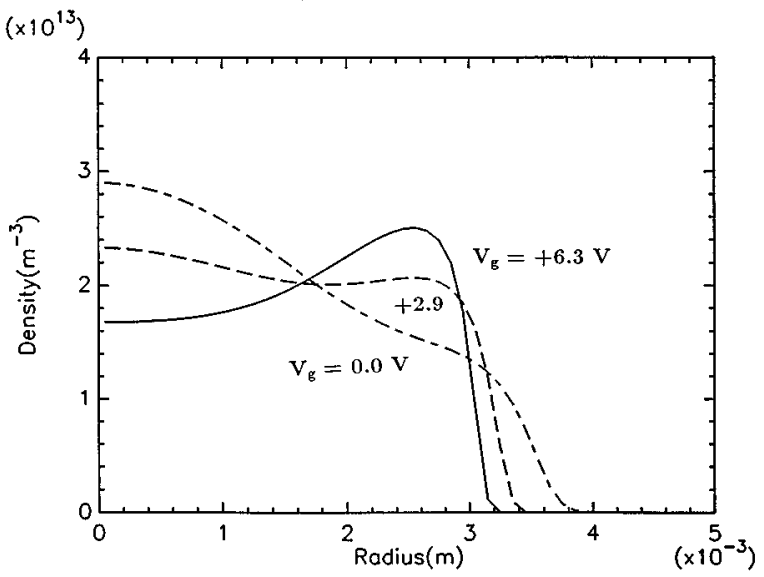

FIG. 7. Midplane density profiles. The profiles shown are for plasmas of longer radial extent $(\alpha=0.0099-0.014)$ where the effect of the nonideal shape of the ring and the effect of the guard ring are more pronounced. The solid curve is for a guard ring voltage of $+6.3 \mathrm{~V}$. The curve for $+2.9 \mathrm{~V}$ is bracketed by the curves for $0.0 \mathrm{~V}$ and $+6.3 \mathrm{~V}$. 
judge the best tuning voltage to be $+6.3 \mathrm{~V}$. Indeed, this was precisely the experimental value of the tuning voltage obtained by Weimer et al. using the local tuning procedure already described in Sec. III. However, if the radius of the plasma began with a radius of about $2.0 \mathrm{~mm}$ and then gradually increased in radius with time, it would extend further and further into a region where the tuning is not ideal. Under these conditions, the actual choice of a guard ring voltage of $+2.9 \mathrm{~V}$ by Weimer et al. ${ }^{10}$ might be a better compromise.

The equilibrium density profiles (here we use global thermal equilibria) which can exist in the trap also depend on the guard ring voltages as shown in Figs. 5, 6, and 7. The three cases shown in Fig. 5 are larger aspect ratio numerical plasmas shown for each of the three guard ring voltages, 0.0 , +2.9 , and $+6.3 \mathrm{~V}$. Figure 6 shows somewhat smaller aspect ratios and Fig. 7 shows a similar plot for the smallest aspect ratios in the series.

For the smallest aspect ratios (larger radial extent) of Fig. 7, the guard ring voltage of $+2.9 \mathrm{~V}$ produces a midplane density profile which is nearly flat-topped and which has a very sharp rolloff near its radial end. Of the three profiles, this is most nearly like what one might expect for a zerotemperature case such as is assumed in the Dubin theory, i.e. flat top and sharp edge. The choice of $+6.3 \mathrm{~V}$ for the guard ring voltage again produces an equilibrium with a sharp edge, although the flat top is noticeably absent. We observe also in the case where the guard ring voltage is $0.0 \mathrm{~V}$, the equilibrium has a fairly diffuse boundary, and it is not so clear exactly how the aspect ratio is to be defined.

In order to investigate the effect of the distortion of radial profiles arising from the non-ideal geometry and thermal effects on the spread of aspect ratio predictions of the Dubin theory, we created numerical equilibria of different aspect ratios and guard ring voltages in our model trap of Fig. 2. These were used as the starting point for PIC simulations of the $m=0$ modes of the trap. The guard ring voltages were taken to be $0.0 \mathrm{~V}$, $+2.9 \mathrm{~V}$, and $+6.3 \mathrm{~V}$. The radii of the plasmas ranged in three steps for each voltage from approximately $2.4 \mathrm{~mm}$ (largest aspect ratio) to approximately 3.2 $\mathrm{mm}$ (smallest aspect ratio). Thus, there were nine separate cases to be compared. Tables I and II summarize the results for nine combinations of guard voltage and radial extent of the plasma. Table I shows the frequencies from which the aspect ratios of Table II are computed using Eqs. (2)-(5). In some instances the spacing between modes was irregular (not the expected slightly monotonically changing spacing with
TABLE I. Frequencies of axisymmetric modes of simulated plasmas. The frequencies shown are for different modes, $(i, 0)$, for given choices of guard potential $\left(V_{g}\right)$ and plasma aspect ratio, $\alpha$, in Fig. 2. These are the "data" produced in the numerical experiments.

\begin{tabular}{cccccc}
\hline \hline $\begin{array}{c}V_{g} \\
(\mathrm{~V})\end{array}$ & $\alpha$ & $(1,0)$ & $\begin{array}{c}(3,0) \\
\left(\times 10^{8}\right.\end{array}$ & $\begin{array}{c}(5,0) \\
\left.\mathrm{s}^{-1}\right)\end{array}$ & $(7,0)$ \\
\hline 0.0 & 0.022 & 3.7910 & 3.7525 & $3.7116^{\mathrm{a}}$ & 3.6702 \\
0.0 & 0.017 & 3.7929 & 3.7677 & $3.7480^{\mathrm{a}}$ & 3.7210 \\
0.0 & 0.014 & 3.7927 & 3.7736 & 3.7543 & $3.7315^{\mathrm{a}}$ \\
2.9 & 0.020 & 3.7896 & 3.7408 & $3.7060^{\mathrm{a}}$ & 3.6572 \\
2.9 & 0.014 & 3.7872 & 3.7525 & 3.7222 & 3.6967 \\
2.9 & 0.011 & 3.7847 & 3.7579 & 3.7340 & 3.7121 \\
6.3 & 0.019 & 3.7912 & 3.7351 & 3.6869 & 3.6520 \\
6.3 & 0.017 & 3.7870 & 3.7472 & 3.7121 & 3.6803 \\
6.3 & 0.0099 & 3.7835 & 3.7566 & 3.7327 & 3.7106 \\
\hline \hline
\end{tabular}

${ }^{\mathrm{a}}$ Some uncertainty in identifying this mode.

increasing mode number) and these frequencies are described as "some uncertainty in identifying mode." These instances are associated with some noisiness in the Fourier transforms of the time sequences used to identify modes.

Referring to Table II, we observe several patterns. The aspect ratios obtained from the Dubin theory [Eqs. (2)-(5)] are lower (by factors ranging from $0.40-0.80$ ) than the actual aspect ratios of the plasmas from which they are derived. The largest discrepancies of this sort are associated with a guard potential of $0.0 \mathrm{~V}$. In this case the plasma has a more diffuse radial boundary than the other cases, one which is least like the flat-topped, sharp-edged profiles used in the zero-temperature theory. If the temperature is elevated from zero to $4 \mathrm{~K}$, the Debye length of the plasmas increases, the axial and radial profiles become more diffuse, and the central density of the plasma decreases. The Dubin formulas are blind to this temperature effect and the result is that the theory predicts aspect ratios that are lower than are correct for a given plasma. As the guard voltage is increased, the profiles tend to have a sharper radial profile and the agreement with the theory improves.

However, the spread of the aspect ratios obtained from the formulas is smallest for a guard potential of $0.0 \mathrm{~V}$ and somewhat larger for guard potentials of $+2.9 \mathrm{~V}$ and $+6.3 \mathrm{~V}$. The spreads in the case of $V_{g}=+2.9 \mathrm{~V}$ (the Weimer experimental condition) range from $10-15 \%$ for different aspect ratios, but we do not see (nor did Weimer et al.) a systematic correlation with aspect ratio, i.e. with the degree to which the plasma protrudes into the region where the Penning fre-

TABLE II. Aspect ratios from Dubin theory for simulated plasmas. Results are shown for different choices of guard potential $\left(V_{g}\right)$ and plasma aspect ratio, $\alpha$, in Fig. 2. Subscripted values $\alpha_{(i, 0)}$ are obtained from the frequencies of Table I using Eqs. (3) $-(5)$. Here $\Delta \alpha=\alpha_{(3,0)}-\alpha_{(7,0)} ; \alpha=\left(\alpha_{(3,0)}+\alpha_{(7,0)}\right) / 2$.

\begin{tabular}{|c|c|c|c|c|c|c|c|c|c|}
\hline & \multicolumn{3}{|c|}{ Largest } & \multicolumn{3}{|c|}{$\begin{array}{c}\text { Medium } \\
V_{g}(\mathrm{~V})\end{array}$} & \multicolumn{3}{|c|}{ Smallest } \\
\hline & 0.0 & 2.9 & 6.3 & 0.0 & 2.9 & 6.3 & 0.0 & 2.9 & 6.3 \\
\hline$\alpha$ & 0.022 & 0.020 & 0.019 & 0.017 & 0.014 & 0.017 & 0.014 & 0.011 & 0.0099 \\
\hline$\alpha_{(3,0)}$ & 0.010 & 0.013 & 0.015 & 0.0068 & 0.0093 & 0.011 & 0.0051 & 0.0072 & 0.0072 \\
\hline$\alpha_{(5,0)}$ & $0.011^{\mathrm{a}}$ & $0.011^{\mathrm{a}}$ & 0.014 & $0.0060^{\mathrm{a}}$ & 0.0087 & 0.010 & 0.0051 & 0.0068 & 0.0068 \\
\hline$\alpha_{(\underline{7}, 0)}$ & 0.011 & 0.012 & 0.012 & 0.0064 & 0.0080 & 0.0095 & $0.0054^{\mathrm{a}}$ & 0.0064 & 0.0065 \\
\hline$\Delta \alpha / \alpha(\%)$ & 2.8 & 10 & 20 & 6.2 & 15 & 12 & 5.4 & 11 & 11 \\
\hline$\alpha_{(3,0)} / \alpha$ & 0.48 & 0.65 & 0.79 & 0.41 & 0.66 & 0.64 & 0.38 & 0.67 & 0.73 \\
\hline
\end{tabular}

${ }^{a}$ Some uncertainty in identifying this mode. 
TABLE III. Plasma parameters from Dubin theory for simulated plasmas. Results are shown for given choices of guard potential $\left(V_{g}\right)$ and plasma aspect ratio $\alpha$ in Fig. 2. Inferred quantities are calculated assuming a spheroidal plasma whose aspect ratio is calculated from Eq. (3) using frequencies from Table I. Comparison is made to parameters that are known for the simulated plasmas of Fig. 2.

\begin{tabular}{lccccccc}
\hline \hline $\begin{array}{l}V_{g} \\
(\mathrm{~V})\end{array}$ & $\alpha$ & $\begin{array}{c}n_{0} \\
\left(10^{13} \mathrm{~m}^{-3}\right)\end{array}$ & $\begin{array}{c}n_{0}(\text { inferred }) \\
\left(10^{13} \mathrm{~m}^{-3}\right)\end{array}$ & $\begin{array}{c}r_{p} \\
(\mathrm{~mm})\end{array}$ & $\begin{array}{c}r_{p} \text { (inferred) } \\
(\mathrm{mm})\end{array}$ & $\begin{array}{c}z_{p} \\
\left(10^{-2} \mathrm{~mm}\right)\end{array}$ & $\begin{array}{c}z_{p}(\text { inferred }) \\
\left(10^{-2} \mathrm{~mm}\right)\end{array}$ \\
\hline 0.0 & 0.022 & 3.74 & 4.59 & 2.29 & 2.79 & 4.93 & 2.87 \\
0.0 & 0.017 & 3.27 & 4.57 & 2.56 & 3.21 & 4.24 & 2.18 \\
0.0 & 0.014 & 2.89 & 4.56 & 2.81 & 3.52 & 3.84 & 1.82 \\
2.9 & 0.020 & 3.63 & 4.61 & 2.32 & 2.57 & 4.70 & 3.37 \\
2.9 & 0.014 & 2.98 & 4.57 & 2.76 & 2.89 & 3.90 & 2.69 \\
2.9 & 0.011 & 2.33 & 4.55 & 3.16 & 3.15 & 3.39 & 2.27 \\
6.3 & 0.019 & 3.50 & 4.62 & 2.32 & 2.45 & 4.47 & 3.70 \\
6.3 & 0.017 & 2.63 & 4.58 & 2.56 & 2.76 & 4.24 & 2.95 \\
6.3 & 0.0099 & 1.68 & 4.55 & 3.06 & 3.15 & 3.02 & 2.28 \\
\hline \hline
\end{tabular}

quency profile dips. The spread is larger than expected on the basis of the precision of the formulas $(2 \%)$, but less than the spread of the Weimer data $(20-25 \%)$.

If the guard ring voltage is $+6.3 \mathrm{~V}$, the plasma of smallest aspect ratio in the series of three has an aspect ratio of 0.0099 (from the equilibrium code). Figure 7 shows that this equilibrium (at least in the radial midplane) is relatively sharp-edged and has close to the same radius as the smallest aspect ratio equilibrium at $+2.9 \mathrm{~V}$. From the PIC simulation and the Dubin formulas we obtain aspect ratios of 0.0072 , 0.0068 , and 0.0065 , which are very nearly the values obtained for the small aspect ratio equilibrium when the guard ring voltage was $+2.9 \mathrm{~V}$. The spread in aspect ratios is $11 \%$. The close agreement between the aspect ratios from the two different cases is reassuring since in both cases the plasmas have relatively sharp and similar radial definition.

If one assumes that the aspect ratio of the simulated plasma is known from applying Eq. (3) to frequencies obtained from the simulations, one may obtain the inferred density of the plasma from Eq. (10). Assuming uniform density and spheroidal shape for which the volume is $V=4 / 3 \pi r_{p}^{2} z_{p}$, one may infer a radius and axial length for the plasma. Since these quantities are also known in advance for the simulated plasma, one may estimate the degree to which these quantities might be obtained if the modelled plasmas resemble experimental conditions. The results of this comparison are shown in Table III.

Table III shows that the densities are overestimated by factors ranging from $1.25-2.70$ if this program is carried out. Note that the comparison is made to the central density of the simulated plasma which is also the maximum density except in those cases where the guard voltage has created a density maximum at the radial extremity of the plasma $\left(V_{g}=+6.3\right.$ V). The estimates worsen as the guard voltage increases and also as the radial extent of the plasma increases. The axial length of the plasma is systematically underestimated, again with the largest discrepancy in the case of vanishing guard voltage. The presence of the guard voltage tends to narrow the axial length of the plasma and compensates somewhat for the diffuse axial boundary of the plasma arising from the non-zero operating temperature. Finally, there is rather remarkable agreement in these instances between the radius of the simulated plasma and the inferred radius, particularly with guard voltages of 2.9 and $6.3 \mathrm{~V}$.

We now shift our attention to temperature as a possible variable. In this study we fixed the guard ring voltage at $+2.9 \mathrm{~V}$ (the operating voltage of the Weimer experiment). We began with the conditions used in generating the $4 \mathrm{~K}$ equilibria used in the study just described, but recomputed the equilibria with the electron temperature elevated to $16 \mathrm{~K}$. Three aspect ratios were again considered. See Table IV.

The effect of the elevated temperature is to leave $\omega_{z}$ unchanged, but to shift the $(3,0),(5,0)$, and $(7,0)$ frequencies upward. The shift is least for $(3,0)$, but is systematically larger for the higher modes. The shift in frequencies is such as to increase the spread of the aspect ratios from $10 \%, 15 \%$, and $11 \%$ in Table II to $14 \%, 30 \%$, and $18 \%$, respectively. These figures do come closer to the experimental spreads reported by Weimer et al. Thus, the spread in experimental aspect ratios could be explained if the electron temperature in the experiments were higher than assumed (by a factor of 4 in our numerical simulations).

\section{CONCLUSIONS}

This work was motivated by the desire to understand the degree to which the theory of Dubin can be applied to Penning trap experiments when the Debye length is comparable to the plasma thickness in realistic geometry. In particular, we hoped to understand better the spread in aspect ratios that results from applying Dubin's theory to the data from Weimer's experiments.

Numerical experiments to correlate the spread in aspect ratios with the proximity of the walls of the trap (induced charge effects) demonstrated that the effect of induced charge is to preferentially shift the center-of-mass mode frequency downward, with systematically decreasing relative shifts downward with increasing mode number. The consequence of these shifts is to reduce the actual discrepancy between the aspect ratio deduced from the $(3,0)$ mode compared to its actual value, but to decrease the spread of values (or even reverse the ordering compared to the experimental data) emerging from Eqs. (2)-(5). Thus our attention was directed increasingly to the effects of realistic geometry in 
TABLE IV. Frequencies of axisymmetric modes of simulated plasmas at 16 $\mathrm{K}$. The results are for the simulated plasmas of Table I with the temperature elevated from $4 \mathrm{~K}$ to $16 \mathrm{~K}$. The guard potential $\left(V_{g}\right)$ is chosen to match the experimental conditions of Weimer et al.

\begin{tabular}{cccccc}
\hline \hline$V_{g}$ & $\alpha$ & $(1,0)$ & $(3,0)$ & $(5,0)$ & $(7,0)$ \\
$(\mathrm{V})$ & & & $\left(\times 10^{8}\right.$ & $\left.\mathrm{s}^{-1}\right)$ & \\
\hline 2.9 & 0.020 & 3.7895 & 3.7448 & & 3.6729 \\
2.9 & 0.014 & 3.7873 & 3.7532 & 3.7274 & 3.7109 \\
2.9 & 0.011 & 3.7852 & 3.7583 & 3.7364 & 3.7179 \\
\hline \hline
\end{tabular}

which the hyperboloid well is only approximated and must be subsequently tuned by a guard ring potential.

Numerical experiments in which the detailed geometry of the trap is simulated, including the presence of a guard ring, indicate that the well potential is distorted in such a way that the vacuum Penning frequency has radial dependence. As the guard ring potential is increased, the distortion increases at larger radii and there is an increase in the spread of aspect ratios that emerges from Eqs. (2)-(5), although in our numerical experiments the spread is lower (10-20\%) than the experimental data of Weimer (20-25\%). The spread is not directly correlated to the radial extent of the plasma, but seems rather to be correlated to distortions in the shape and radial profile of the plasma that occur because the plasma thickness is comparable to the Debye length.

The effect of elevating the temperature in numerical experiments on equilibria in global thermal equilibrium is to increase the spread of the aspect ratios from Eqs. (2)-(5) and is the most likely cause of the experimental spread emerging from this study, although the temperature would have to be a factor four larger than assumed in the experiments. We note that the induced charge effect (primarily reducing the centerof-mass mode frequency) and the effect of increasing temperature (primarily increasing the higher mode frequencies) are countervening effects in determining the aspect ratios via Eqs. (2)-(5). If correction is made for induced charge, the spread in aspect ratios is likely a sharper function of temperature than these simulations and the experiments (which have built-in induced charge effects) demonstrate.

In general, however, we conclude that the Dubin theory does indeed provide a means to obtain reasonable estimates of plasma parameters, including density, radius and axial length, for plasmas even in the very important regime for which the axial length is comparable to a Debye length. In addition, we have identified dependencies on induced charge, equilibrium shape, and plasma temperature which might be used to improve agreement between theory and experiment.

\section{ACKNOWLEDGMENTS}

We gratefully acknowledge helpful communications with John Bollinger and Carl Weimer who provided valuable insight and supplemental information about the experiments to which this work is directed.

\section{APPENDIX: INDUCED CHARGE}

In this appendix we make an estimate of the effects on mode frequencies of induced charge on the conductors shown in Fig. 1. To simplify the calculation, the cylindrical geometry is replaced by an approximate Cartesian geometry where the properties of complex-valued functions can be employed. In this approximate geometry the plasma is modelled as an infinitely long slab of charge with thickness $2 z_{p}$ and width $2 r_{p}$, where $r_{p}$ is the plasma radius and where $z_{p}$ is the half-thickness of the thin plasma. The plasma inside the slab consists of particles of charge $q$, mass $m$, and uniform density $n_{o}$. The effect of the ring-electrode is studied in the geometry shown in part (a) of Fig. 8, while the effect of the endcaps are studied in the geometry of part (b) of the same figure. The quantity $r_{w}$ is the distance from the center of the plasma to the ring electrode, while $z_{w}$ is the distance from the center of the plasma to either endcap. For simplicity, only the results for conductor angle $\beta=0$ will be given in detail. The more general case of non-zero $\beta$ can be handled by the conformal mapping,

$$
w=e^{-i \beta} \zeta^{\gamma} ; \quad \gamma=1+\frac{2 \beta}{\pi},
$$

which maps the complex $w$-plane $(w=x+i y)$ shown in Fig. 8 to the upper-half $\zeta$-plane where an image-charge calculation like the one given below in the $w$-plane can be employed.

\section{Ring electrode}

The motion of the plasma is assumed to be represented by the function $\xi(y, t)$, representing the displacement of the plasma in the direction normal to the thin dimension of the plasma [see part (a) of Fig. 8]. Under such a displacement the perturbed charge density is given by the continuity equation (for a rigid shift),

$$
\delta n=-\xi(y) \frac{\partial n_{o}}{\partial x}=\xi(y) n_{o}\left[\delta\left(x-z_{p}\right)-\delta\left(x+z_{p}\right)\right] .
$$

This perturbed density can be transformed into a perturbed line-charge density $d \lambda$ into the page in the complex plane of Fig. 8 by multiplying it by the charge on a particle and integrating it over a small area $d x d y$. In the $x$-direction the delta functions cause the line charge density only to exist at $\pm z_{p}$ and in the $y$-direction the integral, for small $d y$, is obtained simply by multiplying by $d y$, giving

$$
d \lambda=q n_{o} \xi(y) d y \begin{cases}+1 & \text { at } x=z_{p} \\ -1 & \text { at } x=-z_{p}\end{cases}
$$

We now estimate the effect on the mode frequencies of the slab produced by the induced charge in the ring electrode of this dipole-layer charge distribution. We take an image of the perturbed charge mirrored through the conducting plane along the real axis into the lower half of the complex $w$-plane. The complex electrostatic field produced by this image charge at any point $w$ of the plasma is given by ${ }^{13}$ 


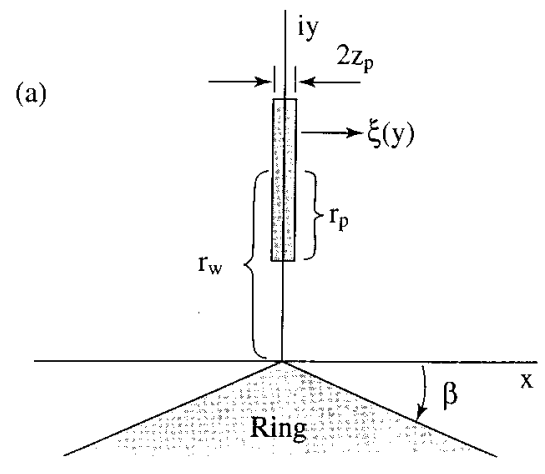

(b)

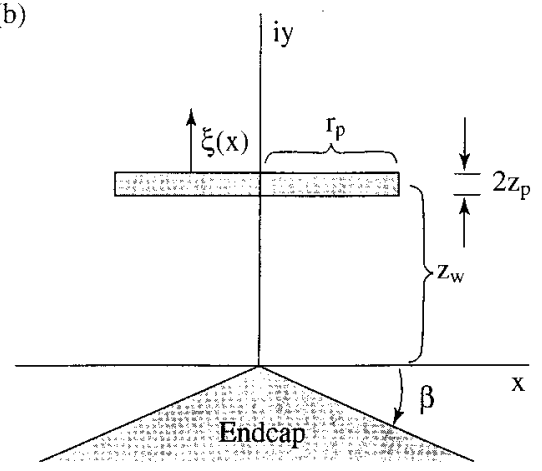

FIG. 8. Slab geometry for induced charge estimates. The Penning trap is approximated by a Cartesian complex plane representation for purposes of estimating the effect on the mode frequencies of induced charge in the ring and endcaps.

$$
\begin{aligned}
\delta E_{x}(w)-i \delta E_{y}(w)= & \int_{r_{w}-r_{p}}^{r_{w}+r_{p} q n_{o} \xi\left(y^{\prime}\right)} \frac{-1}{2 \pi \epsilon_{o}}\left[\frac{-z_{p}+i y^{\prime}}{w}\right. \\
& \left.+\frac{1}{w+z_{p}+i y^{\prime}}\right] d y^{\prime} .
\end{aligned}
$$

In the small aspect ratio limit where $z_{p} \ll r_{p}$ and in the plasma at $w=i y$ where the field of the induced charge is to be evaluated, this expression becomes

$$
\begin{aligned}
\delta E_{x}(y) & \approx \frac{q n_{o} 2 z_{p}}{2 \pi \epsilon_{o}} \int_{r_{w}-r_{p}}^{r_{w}+r_{p}} \frac{d}{d w} \frac{1}{\left(w+i y^{\prime}\right)} \xi\left(y^{\prime}\right) d y^{\prime} \\
& =\frac{q n_{o} z_{p}}{\pi \epsilon_{o}} \int_{r_{w}-r_{p}}^{r_{w}+r_{p}} \frac{\xi\left(y^{\prime}\right) d y^{\prime}}{\left(y+y^{\prime}\right)^{2}} .
\end{aligned}
$$

It should be noted that a better approximation to the cylindrical case could be obtained by using another conducting plane above the plasma in part (a) of Fig. 8. This, however, makes the problem much more complicated. Trying something simple such as doubling the induced electric field does not work for all points in the plasma because points near one wall feel a strong effect from that wall and a weak effect from the other one. Therefore, as an approximation we will just include the effect of one wall, but only use its field over the half of the plasma that is nearest to the lower wall, i.e., for $r_{w}-r_{p} \leqslant y \leqslant r_{w}$.
To estimate the effect of this field on the electrostatic modes of vibration we assume a time dependence of $e^{i \omega t}$ and write the equation of motion in the form

$$
-m \omega^{2} \xi(y)=q \delta E_{x}(y)+\ldots,
$$

where the other terms corresponding to physical effects other than the field of the induced charge have been suppressed. We multiply this equation by the displacement function $\xi(y)$ and integrate it from the center of the plasma to the tip nearest the conducting plane to obtain an estimate of the shift $\delta\left(\omega^{2}\right)$ in $\omega^{2}$ due to induced charge:

$$
\begin{aligned}
\frac{\delta\left(\omega^{2}\right)}{\omega_{p}^{2}}= & -\frac{z_{p}}{\pi \int_{r_{w}-r_{p}}^{r_{w}} \xi(y)^{2} d y} \\
& \times \int_{r_{w}-r_{p}}^{r_{w}} d y \int_{r_{w}-r_{p}}^{r_{w}+r_{p}} d y^{\prime} \frac{\xi\left(y^{\prime}\right) \xi(y)}{\left(y+y^{\prime}\right)^{2}},
\end{aligned}
$$

where $\omega_{p}$ is the plasma frequency in the thin cloud. When the displacement function $\xi(y)$ is taken to be one of Dubin's displacement functions for the $(3,0)$ mode, or higher, the frequency shifts are found to be an order of magnitude less than the frequency shift for the center of mass mode, for which $\xi$ is uniform in space. If the displacement is constant, the integral can be performed to obtain for the center of mass mode,

$$
\frac{\delta\left(\omega^{2}\right)}{\omega_{p}^{2}}=-\frac{\alpha}{\pi} \ln \left[\frac{\left(2 r_{w}-r_{p}\right) r_{w}}{\left(2 r_{w}+r_{p}\right)\left(r_{w}-r_{p}\right)}\right],
$$

where $\alpha$ is the aspect ratio $\alpha=r_{p} / z_{p}$.

We note in passing that the effect of making the angle $\beta$ non-zero is to reduce the frequency shifts. This calculation is a bit complicated, but to within $10-20 \%$ the effect of finite angle is to reduce $\delta\left(\omega^{2}\right) / \omega_{p}^{2}$ by the factor $\gamma^{2}$, where $\gamma=1+2 \beta / \pi$.

\section{Endcaps}

For the geometry of part (b) of Fig. 8 with $\beta=0$, we have

$$
\delta n=-\xi(x) \frac{\partial n_{o}}{\partial y}
$$

and

$$
d \lambda=q n_{o} \xi(x) d x \begin{cases}+1, & \text { at } y=z_{w}+z_{p}, \\ -1, & \text { at } x=z_{w}-z_{p} .\end{cases}
$$

With the image charge again mirrored into the lower half $w$-plane by a conducting plane along the real axis, we have

$$
\begin{aligned}
\delta E_{x}(w)-i \delta E_{y}(w)= & 2 \times \int_{-r_{p}}^{r_{p}} \frac{q n_{o} \xi\left(x^{\prime}\right)}{2 \pi \epsilon_{o}} \\
& \times\left[\frac{-1}{w-x^{\prime}+i\left(z_{w}+z_{p}\right)}\right. \\
& \left.+\frac{1}{w-x^{\prime}+i\left(z_{w}-z_{p}\right)}\right] d x^{\prime} .
\end{aligned}
$$


The factor of two multiplying the integral approximately takes into account the presence of two endcaps on which there is induced charge, with the plasma midway between them (see Fig. 1). In the small aspect ratio limit where $z_{p} \ll r_{p}$ and in the plasma at $w=x+i z_{w}$ where the field of the induced charge is to be evaluated we obtain for the $y$-component of the field

$$
\delta E_{y}(x) \approx-\frac{2 q n_{o} z_{p}}{\pi \epsilon_{o}} \operatorname{Re} \int_{-r_{p}}^{r_{p}} \frac{\xi\left(x^{\prime}\right) d x^{\prime}}{\left(x-x^{\prime}+2 i z_{w}\right)^{2}},
$$

where $R e$ denotes the real part.

Just as was done with the ring electrode, the equation of motion is used to obtain an approximate form for the frequency shift:

$\frac{\delta\left(\omega^{2}\right)}{\omega_{p}^{2}}=\frac{2 z_{p}}{\pi \int_{-r_{p}}^{r_{p}} \xi(x)^{2} d x} \int_{-r_{p}}^{r_{p}} d x \int_{-r_{p}}^{r_{p}} d x^{\prime} \frac{\xi\left(x^{\prime}\right) \xi(x)}{\left(x-x^{\prime}+2 i z_{w}\right)^{2}}$.

(Note that the imaginary part of this double integral is zero, so it is no longer necessary to take the real part.) As in the ring electrode case, the frequency shifts for the $(3,0)$ and higher modes are about an order of magnitude smaller than the shift for the center of mass mode. Since the center of mass mode has a constant displacement function, we obtain for the frequency shift of this mode due to the endcaps,

$$
\frac{\delta\left(\omega^{2}\right)}{\omega_{p}^{2}}=-\frac{\alpha}{\pi} \ln \left(1+\frac{r_{p}^{2}}{z_{w}^{2}}\right)
$$

In this case the effect of making the angle $\beta$ non-zero is also to reduce the frequency shifts, approximately by the factor $\gamma$.

Equations (A8) and (A14) have been tested with the particle simulation for a few choices of plasma sizes and wall positions, and appear to be low by about a factor of two for the center-of-mass mode. The simulations also confirm that the shifts in the higher modes are negligible. Adding an empirical correction factor of two and combining the effects from ring electrode and endcaps gives the following approximate formula for the frequency shift of the center-of-mass mode:

$$
\begin{aligned}
\frac{\delta \omega}{\omega_{p}} \approx & -\frac{\alpha}{\pi}\left\{\frac{1}{\gamma_{\text {ring }}^{2}} \ln \left[\frac{\left(2 r_{w}-r_{p}\right) r_{w}}{\left(2 r_{w}+r_{p}\right)\left(r_{w}-r_{p}\right)}\right]\right. \\
& \left.+\frac{1}{\gamma_{\text {end }}} \ln \left[1+\frac{r_{p}^{2}}{z_{w}^{2}}\right]\right\},
\end{aligned}
$$

where $\gamma_{\text {ring }}$ and $\gamma_{\text {end }}$ are the values of $\gamma$ appropriate for the ring electrode and the endcaps and where the empirical factor of two has disappeared because this formula gives $\delta \omega / \omega_{p}$ instead of $\delta\left(\omega^{2}\right) / \omega_{p}^{2}$. The corresponding simple approximation for the frequency shift of the higher modes is that these shifts are zero.
Hence, the effect of induced charge on nearby conductors is to decrease the frequency of the center-of-mass mode while leaving the other frequencies mostly unchanged. The effect of this shift on the values of $\alpha$ inferred from the spheroid formulas [Eqs. (2)-(5)] is to decrease all of the $\alpha$-values, but to decrease $\alpha_{(3,0)}$ the most, $\alpha_{(5,0)}$ by less, $\alpha_{(7,0)}$ by even less, and so on. This causes the spheroid formulas to underestimate the true value of $\alpha$ and also to cause spreading of the predicted $\alpha$-values, with higher modes giving higher values of $\alpha$, opposite to the spreading observed in the experiments of Weimer et al. This means that if the effects of image charge are removed from the experimental data, the spreading in $\alpha$ would be even larger than it already is.

As an example of this effect, consider the frequency data for guard-ring voltages $V_{g}=2.9 \mathrm{~V}$ in Table I. We can approximately correct this data for the effect of image charge by raising the frequency of the center-of-mass mode by the amount given by the approximate formula Eq. (A15). In doing so we use density values for the actual equilibria obtained from Table III, $r_{w}=5 \mathrm{~mm}, z_{w}=3.5 \mathrm{~mm}$, $\beta_{\text {ring }}=0.36$, and $\beta_{\text {end }}=0.15$, giving $\gamma_{\text {ring }}=1.2$ and $\gamma_{\text {end }}=1.1$. The values of the angles $\beta_{\text {end }}$ and $\beta_{\text {ring }}$ are taken from Fig. 2. For each of the three cases considered, we take the actual values of $\alpha$ and $r_{p}$ from Tables II and III. The result of doing this correction is to decrease the discrepancy between the actual value of $\alpha$ and the value inferred from the $(3,0)$ mode by about $50 \%$ and to roughly double the spread between the $\alpha$-values inferred from the $(3,0)$ and $(7,0)$ modes. For example, for $V_{g}=2.9 \mathrm{~V}$ and in the medium case ( $\alpha=0.014$ ) we find $\alpha_{(3,0)}=0.011$ and $\alpha_{(7,0)}=0.00856$. Since the experimental data of Weimer et al. were affected by nearby conductors in the same way as the simulation, their computed values of $\alpha_{(3,0)}$ would also be closer to the true values, and the spread in their data would be larger if their frequencies were corrected for the effects of induced charge.

${ }^{1}$ G. Gabrielse, D. Phillips, W. Quint, H. Kalinowsky, G. Rouleau, and W. Jhe, Phys. Rev. Lett. 74, 3544 (1995)

${ }^{2}$ R. G. Greaves and C. M. Surko, Phys. Rev. Lett. 75, 3846 (1995).

${ }^{3}$ An older review of applications is provided in F. L. Walls and G. H. Dunn, Phys. Today 27, 30 (1974); see also C. S. Weimer, J. J. Bollinger, F. L. Moore, and D. J. Wineland, Phys. Rev. A 49, 3842 (1994).

${ }^{4}$ D. H. E. Dubin, Phys. Rev. Lett. 66, 2076 (1991).

${ }^{5}$ M. D. Tinkle, R. G. Greaves, C. M. Surko, R. L. Spencer, and G. W. Mason, Phys. Rev. Lett. 40, 352 (1994).

${ }^{6}$ C. S. Weimer, J. J. Bollinger, F. L. Moore, and D. J. Wineland, Phys. Rev. A 49, 3842 (1994).

${ }^{7}$ J. J. Bollinger, D. J. Heinzen, F. L. Moore, W. M. Itano, D. J. Wineland, and D. H. E. Dubin, Phys. Rev. A 48, 525 (1993).

${ }^{8}$ D. J. Heinzen, J. J. Bollinger, F. L. Moore, Wayne M. Itano, and D. J. Wineland, Phys. Rev. Lett. 66, 2080 (1991).

${ }^{9}$ T. M. O’Neil, Phys. Fluids 23, 2216 (1980).

${ }^{10}$ J. J. Bollinger (private communication, 1995).

${ }^{11}$ R. L. Spencer, S. N. Rasband, and R. R. Vanfleet, Phys. Fluids B 5, 4267 (1993).

${ }^{12}$ S. A. Prasad and T. M. O’Neil, Phys. Fluids 22, 278 (1979).

${ }^{13}$ W. R. Smythe, Static and Dynamic Electricity (McGraw-Hill, New York, 1939), Chap. 4. 